1987, 36,4

УдК 519.24

В. ИОАЛА, В. ОЛЬМАН

\title{
МЕТОД ОЦЕНИВАНИЯ КОЭФФИЦИЕНТОВ ЛИНЕЙОЙ ОДНОМЕРНОЙ РЕГРЕССИИ
}

v. JOALA, $v$. OLMAN. LINEAARSE OHEMOOTMELISE REGRESSIOONI KOEFITSIENTIDE HINDAMISE MEETOD

v. JOALA, V. OLMAN. AN ESTIMATION METHOD OF COEFFICIENTS OF ONE-DIMENSIONAL LINEAR REGRESSION

$$
\text { (Представил Н. Алумяэ) }
$$

Пусть наблюдается процесс

$$
x(t)=a_{0}+a_{1} t+u_{t}, \quad t=1, \ldots, M,
$$

где случайные величины $u_{t}$ некоррелированы и имеют средние значения $E u_{t}=0$ и дисперсии $E u_{t}^{2}=\sigma^{2}>0$.

Для оценивания коэффициентов $a_{0}$ и $a_{1}$ обычно применяют метод наименьших квадратов [ $\left.{ }^{1}\right]$. С этой целью минимизируется по $a_{0}$ и $a_{1}$ следующая функция

$$
\sum_{t=1}^{M} u_{t}^{2}=\sum_{t=1}^{M}\left[x(t)-a_{0}-a_{1} t\right]^{2} .
$$

Оценки $\bar{a}_{0}$ и $\bar{a}_{1}$ коэффициентов $a_{0}$ и $a_{1}$ определяются следующим образом:

$$
\begin{aligned}
& \vec{a}_{1}=\frac{12 \sum_{t=1}^{M} t x(t)-6(M+1) \sum_{t=1}^{M} x(t)}{M\left(M^{2}-1\right)}, \\
& \vec{a}_{0}=\frac{1}{M} \sum_{t=1}^{M} x(t)-\bar{a}_{1} \frac{M+1}{2} .
\end{aligned}
$$

В литературе $\left[{ }^{2,3}\right]$ приведены формулы для определения дисперсий оценок $\bar{a}_{0}$ и $\bar{a}_{1}$.

$$
\begin{aligned}
& D\left(\bar{a}_{1}\right)=\frac{\sigma^{2}}{\sum_{t=1}^{M}(t-\bar{t})^{2}}=\frac{12}{M^{3}-M} \sigma^{2}, \\
& D\left(\bar{a}_{0}\right)=\frac{\sigma^{2}}{M}+(\bar{t})^{2} D\left(\bar{a}_{1}\right)=2 \frac{2 M+1}{M(M-1)} \sigma^{2},
\end{aligned}
$$

где $\bar{t}=\frac{1}{M} \sum_{t=1}^{M} t=\frac{M+1}{2}$, 
Как видно из формул (1) и $(2)$, для вычисления оценок $\vec{a}_{0}$ и $\vec{a}_{1}$ необходимо вычислить суммы

$$
\sum_{t=1}^{M} x(t) \quad \text { и } \quad \sum_{t=1}^{M} t x(t)
$$

которые требуют выполнения $M$ умножений и $2 M$ сложений.

Оценка метода наименьших квадратов является наилучшей среди нелинейных несмещенных оценок. В настоящей работе приведен линейный метод оценивания, который при незначительной потере в точности позволяет существенно сократить время вычислений оценок.

Метод основан на вычислении последовательных разностей элементов исходного ряда с шагом $N$. Для оценивания коэффициента $a_{1}$ усредняется $k$ разностей $(k>0)$

$$
x(t+N)-x(t), \quad t=1, \ldots, k .
$$

I. Оценка

$$
\hat{a_{1}}=\frac{1}{N k} \sum_{t=1}^{k}[x(t+N)-x(t)]=\frac{1}{N k}\left[\sum_{t=N+1}^{N+k} x(t)-\sum_{t=1}^{k} x(t)\right]
$$

является несмещенной и состоятельной оценкой коэффициента $a_{1}$ для любых $N$ и $k(N+k \leqslant M)$.

Д ок а з а т е л в с т в о. Представим оценку $\hat{a}_{1}$ в виде

$$
\hat{a}_{1}=a_{1}+\frac{1}{N k}\left[\sum_{t=1}^{k} u_{t+N}-\sum_{t=1}^{k} u_{t}\right] \text {, }
$$

откуда легко следует, что

$$
E\left(\hat{a}_{1}\right)=a_{1} .
$$

Дисперсию оценки $\hat{a}_{1}$ выведем в двух случаях.

1) При $N+1>k$ получаем

$$
E\left(\hat{a}_{1}-a_{1}\right)^{2}=\frac{1}{N^{2} k^{2}} E\left\{\sum_{t=1}^{k}\left(u_{t+N}-u_{t}\right)\right\}^{2}=\frac{2}{N^{2} k} \sigma^{2} .
$$

Можно показать, что минимальное значение полученной дисперсии при ограничении $N+k \leqslant M$ достигается при $k=M / 3$.

2) В результате несложных преобразований получаем, что при $N+1<k$

$$
E\left(\hat{a}_{1}-a_{1}\right)^{2}=\frac{2}{N k^{2}} \sigma^{2}
$$

Минимум полученной дисперсии достигается здесь при $k=\frac{2 M}{3}$.

При $N+k=M$ ввиду минимизации вычислений выбираем первый вариант $(k=M / 3$ и $N=2 M / 3)$ и отсюда получаем

$$
\min _{N, k} D\left(\hat{a}_{1}\right)=\frac{13,5}{M^{3}} \sigma^{2} .
$$

II. Оценка

$$
\hat{a}_{0}=\frac{1}{M}\left[\sum_{t=1}^{M} x(t)-\hat{a}_{1} \sum_{t=1}^{M} t\right]
$$

является несмещенной и состоятельной оценкой коэффициента $a_{0}$. 
Доказательство.

$$
\begin{aligned}
& \hat{a_{0}}=a_{0}+\frac{1}{M}\left\{\sum_{t=1}^{M}\left[u_{t}-\frac{t}{N k}\left(\sum_{t=1}^{h} u_{t+N}-\sum_{t=1}^{h} u_{t}\right)\right]\right\}, \\
& E\left(\hat{a}_{0}\right)=a_{0}, \\
& E\left(\hat{a}_{0}-a_{0}\right)^{2}=\frac{35 M^{2}+54 M+27}{8 M^{3}} \sigma^{2} .
\end{aligned}
$$

Окончательные формулы вычисления оценок $\hat{a}_{0}$ и $\hat{a}_{1}$ следующие:

$$
\begin{aligned}
& \hat{a_{1}}=\frac{4,5}{M^{2}}\left(S_{3}-S_{1}\right), \\
& \hat{a}_{0}=\frac{1}{M}\left(S_{1}+S_{2}+S_{3}-\hat{a}_{1} \frac{M(M+1)}{2}\right),
\end{aligned}
$$

где $S_{1}=\sum_{t=1}^{k} x(t), \quad S_{2}=\sum_{t=k+1}^{M-k} x(t) \quad$ и $\quad S_{3}=\sum_{t=M-k+1}^{M} x(t)$,

а $k$ ближайшее целое число от $M / 3$.

По приведенным в [ $\left.{ }^{4}\right]$ данным, вычисление оценок $\hat{a}_{10}$ и $\hat{a}_{1}$ по формулам (3) и (4) при использовании арифметики с плавающей запятой на вычислительных машинах CM-3 и CM-4 занимает примерно в 3,2 раза меньше времени, чем вычисление этих оценок по формулам (1) и (2).

Таким образом, показано, что дисперсии оценок предлагаемого метода имеют такой же порядок по $M$, как и дисперсии оценок метода наименьших квадратов, и что при существенном сокращении времени вычислений, точность уменьшается незначительно, т. е. оценки (3) и (4) имеют при $M \rightarrow \infty$ соответственно на 12,5 и $9,4 \%$ большую дисперсию, чем оценки метода наименьших квадратов (1) и (2).

\section{Л ИТЕРА Т У Р А}

1. Андерсон T. Статистический анализ временных рядов. М., «Мир», 1976.

2. Бендат Дж., Пирсол А. Измерение и анализ случайных процессов. М., «Мир», 1971.

3. Джонсон $H$., Лион Ф. Статистика и планирование эксперимента в технике и науке. Методы обработки данных. М., «Мир», 1980.

4. Малые ЭВМ и их применение (ред. Б. Н. Наумов). М., «Статистика», 1980.

Институт кибернетики Академии наук Эстонской ССР
Поступила в редакцию 2/III 1987 